\title{
Activité anti-inflammatoire du décocté aqueux des écorces de racines de Morinda geminata DC (Rubiaceae)
}

\author{
Blaise Niankoye BOOLAMOU ${ }^{1}$, Allister Rock LAPO ${ }^{2}$, Kalil Polia CAMARA ${ }^{3}$, \\ Moussa ASSANE ${ }^{2}$, Emmanuel BASSENE $^{1 *}$ et Abdoulaye SAMB ${ }^{4}$ \\ ${ }^{1}$ Laboratoire de Pharmacognosie et Botanique (FMPO, UCAD), Dakar, Sénégal. \\ ${ }^{2}$ Laboratoire de Physiologie, de Pharmacodynamique et de Thérapeutique de l'Ecole \\ Inter-Etats des Sciences et de Médecine Vétérinaire (EISMV, UCAD), Dakar, Sénégal. \\ ${ }^{3}$ Laboratoire de Microbiologie (FSN, UJNK) Kankan, R.G. \\ ${ }^{4}$ Laboratoire de Chimie des Produits Naturels (FST, UCAD), Dakar, Sénégal. \\ *Auteur correspondant ; E-mail : aynenut@gmail.com
}

\section{RESUME}

La présente étude a pour but d'évaluer l'activité anti-inflammatoire de Morinda geminata $D C$, plante de la famille des Rubiaceae largement répandue dans la sous-région d'Afrique de l'Ouest. Des rats de souche wistar ont été répartis en 2 lots de 5 puis gavés aux extraits aqueux de racines de Morinda geminata $\mathrm{DC}$ aux doses de $100 \mathrm{mg} / \mathrm{kg}(\mathrm{n}=5)$ et $300 \mathrm{mg} / \mathrm{kg}(\mathrm{n}=5)$. Ils ont ensuite été soumis à l'épreuve d'œdème induit par la carragénine. Au bout de 3 heures, les effets de la plante ont été comparés avec ceux d'animaux témoins recevant de l'eau distillée $(\mathrm{n}=5)$ et à ceux recevant de l'aspirine $10 \mathrm{mg} / \mathrm{kg}(\mathrm{n}=5)$ comme anti-inflammatoire de référence. 3 heures après traitement, les pourcentages d'inhibition de l'œdème sont de 46,23\%, 37,49\% et 44,13\% respectivement pour les lots Aspirine, Morinda $100 \mathrm{mg}$ et Morinda $300 \mathrm{mg}$. Les résultats des mesures dans tous les lots, l'effet maximal est obtenu au bout de 2 heures. Ces résultats permettent d'affirmer que les extraits aqueux de Morinda geminata $D C$ expriment une activité antiinflammatoire.

(C) 2014 International Formulae Group. All rights reserved.

Mots clés: Morinda geminata DC, anti-inflammatoire, rat.

\section{INTRODUCTION}

Morinda geminata DC est un arbuste à rameaux quadrangulaires pouvant atteindre 10 à $15 \mathrm{~m}$ de hauteur et à glomérules de fleurs blanches odorantes. On le rencontre principalement en Haute et Basse Guinée dans les terrains humides de même qu'en Guinée forestière (Carrière, 1994). Il se rencontre également dans les forêts secondaires ou dans les jachères en Casamance au Sénégal, surtout le long du littoral et la région dakaroise, en Gambie, en Sierra Leone, au Mali, en Côte d'Ivoire, au Nigeria et au Cameroun. Sur le plan chimique, les recherches antérieures signalent l'absence de flavonoïdes, de saponosides, de tanins et d'alcaloïdes dans la plante (Pousset 1989; Kerharo, 1974). Par contre, la présence des quinones dans les écorces de tronc et de racines a été signalée en quantité élevée. Le genre Morinda geminata 
DC était surtout connu pour ses propriétés tinctoriales. Sur le plan de la pharmacopée, la plante est utilisée contre les œdèmes, les entéralgies, les fièvres, les ictères, l'hémoglobinurie bilieuse et la toux (Nalcouma, 1996). L'objectif de ce travail est d'évaluer l'activité anti-inflammatoire des extraits d'écorces de racines sur des rats de laboratoire.

\section{MATERIEL ET METHODES Matériel végétal}

Les écorces de racines ont été récoltées à Koropara-centre (N'Zérékoré) en République de Guinée, au mois de Juillet 2011. Elles ont été séchées à l'abri du soleil. Les écorces ont été broyées au laboratoire de Pharmacognosie de la Faculté de Médecine Pharmacie Odontostomatologie de l'Université Cheikh Anta DIOP (UCAD) Dakar Sénégal.

\section{Matériel animal}

L'étude a porté sur 20 rats mâles wistar, de poids variant entre $125 \mathrm{~g}$ et $250 \mathrm{~g}$, provenant de l'animalerie du laboratoire de Physiologie, Pharmacodynamique et Thérapeutique de l'Ecole Inter-Etats des Sciences et de Médecine Vétérinaire (EISMV/ UCAD) de Dakar Sénégal.

\section{Autres matériels}

Une solution de Carragenine $1 \%$ a servit pour induit l'œdème aux rats. L'Aspirine à $500 \mathrm{mg}$ été administré aux rats du lot référence à la dose de $10 \mathrm{mg} / \mathrm{kg}$. Le lot témoin a reçu du liquide physiologique. Le volume des pattes de rat a été mesuré à l'aide d'un pléthysmomètre.

\section{Extraction}

Trois cent trente grammes de poudre d'écorces des racines de Morinda geminata DC ont été portés à ébullition dans 2 litres d'eau distillée pendant $30 \mathrm{mn}$. Le décocté, une fois refroidi, a été filtré puis évaporé à sec. Une quantité de 45,94 g d'extrait sec a été obtenue, soit un rendement à l'extraction de $13,92 \%$.

\section{Détermination des lots}

Au début de l'expérience, le volume de la patte postérieure gauche de chaque animal a été déterminé au moyen d'un pléthysmomètre. Les animaux ont ensuite été répartis en 4 lots de 5 :

Le lot témoin recevait de l'eau distillée. $\mathrm{Au}$ lot référence, on a administré de l'aspirine à la dose de $10 \mathrm{mg} / \mathrm{kg}$ de poids vif corporel. Au Lot 1 Morinda geminata, on a administré l'extrait à la dose de $100 \mathrm{mg} / \mathrm{kg}$ de poids vif corporel. Le Lot 2 Morinda geminata a reçu l'extrait à la dose de $300 \mathrm{mg} / \mathrm{kg}$ de poids vif corporel.

L'administration des différentes solutions a été réalisée par gavage à l'aide d'une sonde bucco-œsophagienne. Le volume administré à chaque animal était de $1 \mathrm{ml}$.

\section{Induction de l'œdème}

La méthode décrite par Dieng (2013) a été utilisée. Celle-ci consiste à injecter au niveau de l'aponévrose plantaire de la patte postérieure gauche de l'animal 0,05 $\mathrm{ml}$ d'une suspension de carragénine à $1 \%$. Les animaux sont ensuite laissés dans des cages pendant 30 minutes jusqu'à apparition d'un œdème franc.

\section{Evaluation des effets anti-inflammatoires}

Trois heures après l'induction de l'œdème, le développement de l'inflammation a été évalué au moyen du pléthysmomètre, toutes les 60 minutes pendant 3 heures. Les mesures des volumes avant et après administration de la carragénine ont permis de déterminer le pourcentage d'inhibition de l'œdème : d'après la formule : \% d'inhibition $=\left[\mathrm{V}_{2} \text { lot traité- } \mathrm{V}_{1} \text { lot traité }\right]^{*} 100 /\left[\mathrm{V}_{2}\right.$ témoin $-\mathrm{V}_{1}$ témoin]

$\mathrm{V}_{2}$ : Volume de la patte après administration de carragénine

$\mathrm{V}_{1}$ : volume de la patte avant administration de carragénine

\section{Analyse statistique \\ Les résultats sont présentés sous forme de moyenne \pm écart-type. La comparaison entre les lots a été faite par le test}


$t$ de Student. Les valeurs de $\mathrm{p}<0,05$ sont considérées comme significatives.

\section{RESULTATS}

Évolution du volume de l'œdème en fonction du temps

L'évolution de l'œdème en fonction du temps est illustrée par la Figure 1. Celle-ci montre que l'œdème augmente progressivement dans tous les lots, jusqu'à T2 (120 minutes), moment à partir duquel il commence à régresser.

\section{Volume de l'odème}

Le volume moyen de l'œdème obtenu en fin d'expérience et le pourcentage d'inhibition sont présentés dans le Tableau 1. Les résultats font apparaître que l'œdème est plus important dans le lot témoin. Les traitements à l'aspirine et au Morinda geminata à $100 \mathrm{mg} / \mathrm{kg}$ et à $300 \mathrm{mg} / \mathrm{kg}$ ont entrainé une inhibition de l'œdème respectivement de $46,23 \%, 37,49 \%$ et 44 , $13 \%, 3$ heures après traitement. L'analyse statistique montre que la différence entre les lots traités et l'aspirine n'est pas significative ; par contre elle est significative entre les lots traités $(\mathrm{p}=0,0004)$.

Tableau 1 : Volume moyen de l'œdème et pourcentage d'inhibition après 3 heures.

\begin{tabular}{ccc}
\hline Lots & $\begin{array}{c}\text { vol moyen de l'œdème } \\
(\mathbf{m l})\end{array}$ & \% inhibition \\
\hline Témoin & $1,23 \pm 0,39$ & 0 \\
Aspirine & $0,94 \pm 0,40^{\mathrm{ab}}$ & 57,34 \\
Morinda 100 & $0,92 \pm 0,20^{\mathrm{a}}$ & 60,73 \\
Morinda 300 & $0,92 \pm 0,14^{\mathrm{b}}$ & 57,06
\end{tabular}

\footnotetext{
Sur une même colonne, les moyennes suivies d'une lettre différente sont significativement différentes au seuil $\mathrm{p}<0,05$
}

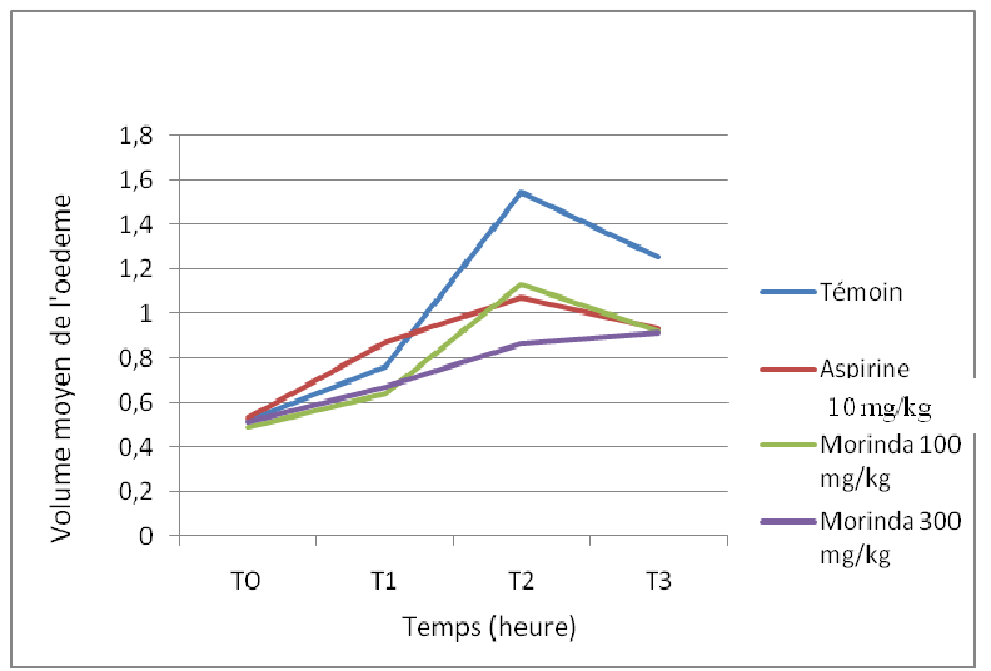

Figure 1 : Evolution de l'œdème dans les différents lots en fonction du temps. $\mathrm{T}_{0}=30 \mathrm{mn} ; \mathrm{T}_{1}=60 \mathrm{mn}$; $\mathrm{T}_{2}=120 \mathrm{mn}$ et $\mathrm{T}_{3}=180 \mathrm{mn}$. 


\section{DISCUSSION}

Nos résultats montrent que la carragénine a provoqué un processus inflammatoire au niveau des pattes des rats. Ce processus inflammatoire s'est traduit par une rougeur et surtout un œdème dont l'effet maximal s'observe en 2 heures. A la fin de l'expérience c'est-à-dire au bout de 3 heures, l'œdème était plus développé dans le lot témoin que dans les lots traités à l'aspirine et aux extraits de Morinda geminata $D C$, où il a été observé une réduction significative.

La carragénine est un agent phlogogène qui est souvent utilisé dans les modèles d'études sur les anti-inflammatoires (Fall, 2009). Son administration provoque un processus inflammatoire caractérisé par la douleur, la chaleur, la rougeur et surtout par le gonflement de la patte postérieure du rat c'està-dire l'œdème.

L'analyse de l'évolution de l'œdème dans le lot témoin montre que celui-ci se développe progressivement jusqu'à atteindre son maximum au bout de 2 heures, moment à partir duquel il commence à régresser.

Bien que ce lot n'ait pas reçu d'antiinflammatoire, il existe au niveau de l'organisme des mécanismes de défense qui permettent de contenir le développement d'un processus inflammatoire. En effet, l'inflammation est caractérisée par une phase vasculaire qui est sous la dépendance de nombreux médiateurs (histamine, sérotonine, quinines, thromboxanes et Platelet activating factor...), et une phase cellulaire au cours de laquelle de nombreuses cellules notamment les polynucléaires neutrophiles et les monocytes envahissent le foyer inflammatoire (Funk, 2001 ; Prescott, 2003). Ces phases se traduisent cliniquement par l'œdème, la rougeur, la douleur et la chaleur. Au-delà de ces phases, survient une phase de réparation dont la finalité est de réparer ou de remplacer le tissu lésé. On peut alors observer une réduction de l'œdème voire la cicatrisation (Miossec, 2003).. C'est ce qui pourrait justifier qu'après 2 heures, l'œdème tend à régresser aussi bien dans le lot témoin que dans les lots traités. Cependant, comme le volume de l'œdème est plus faible dans les lots traités, cela laisse suggérer que tout comme l'aspirine, les extraits de Morinda geminata DC manifestent une activité antiinflammatoire qui vient contrer la réaction inflammatoire normale. En effet, les antiinflammatoires tels que l'aspirine ont la propriété de bloquer la synthèse des prostaglandines issues du métabolisme de l'acide arachidonique d'origine membranaire. (Gogny, 1992).

Dans les conditions de l'expérience, on peut affirmer que les extraits aqueux des écorces de racines de Morinda geminata DC possèdent une activité anti-inflammatoire comparable à celle de l'aspirine.

Cette activité anti-inflammatoire a été retrouvée dans des extraits de plusieurs plantes dont Borassus aethiopum. En effet Sankande (2004) puis plus tard Dieng (2013) ont démontré une activité anti-inflammatoire des extraits aqueux et fractions de Borassus aethiopum. Ces auteurs ont par contre attribué cette activité à la présence de flavonoïdes et de tanins dans les extraits, composés supposés absents des extraits de Morinda geminata DC. Les principes actifs responsables de cette activité seront donc recherchés.

\section{REFERENCES}

Carrière M. 1994. Plantes de Guinée à l'Usage des Eleveurs et des Vétérinaires. Minist. Coop. Fr., CIRAD-EMVT : Paris ; 88.

Crespeau F. 1992. Caractères morphologiques de l'inflammation. Rec. Méd. Vét., 168 (8/9): 545-558.

Dieng M, Fall AD, Sarr SO, Diatta K, Diatta W, Sy GY, Bassene E. 2013. Activité anti-inflammatoire des feuilles de Borassus aethiopum Mart. (Arecaceae). Int. J. Biol. Chem. Sci., 7(2): 805-808.

Fall AD, Sy GY, Fall M, Diatta W, Nicolas JP, Diatta K, Gassama AS, Bassene E, Faye B. 2009. Étude des activités antalgique et anti-inflammatoire et de la toxicité aigüe des feuilles de Aphania senegalensis (Juss. Ex Poir.) Radlk, 
Sapindaceae. Ethnopharmacologia, 43: 76-80.

Funk CD. 2001. Prostaglandins and leukotrienes: advances in eicosanoid biology. Sci., 294: 1871-1875.

Gogny M, Puyt JD. 1992. Activité thérapeutique des anti-inflammatoires non-stéroïdiens. Rec. Méd. Vét., 168 (8/9) : 603-608.

Kerharo J, Adam JG. 1974. La Pharmacopée Sénégalaise Traditionnelle. Plantes Médicinales et Toxiques. Edition Vigot et Frères : Paris.

Koroma L, NITA B. 2009. Phytochemical compounds and antimicrobial activity of three medicinal plants (Alchornea hirtella, Morinda geminata and Craterispermum laurinum) from Sierra Leone. African Journal of Biotechnology, 8(22): 6397-6401.

Miossec P. 2003. Physiopathologie de l'inflammation. Rev. Prat., 53(5): 482488.
Nacoulma GO. 1996. Plantes médicinales et pratique médicale traditionnelle au Burkina Faso, cas du plateau central, Tomes I, II, Doctorat ès Sciences Naturelle. Université de Ouagadougou, p 605.

Pousset JL. 1989. Plantes Médicinales Africaines. Utilisations Pratiques. ACCT, Ellipes: Paris.

Prescott SM, Zimmerman GA, Stafforini D M, McIntyre TM. 2000. Plateletactivating factor and related lipid mediators. Annu. Rev. Bioch., 69: 419445.

Sakande J. 2004. Etude bio-guidée des propriétés pharmacologiques d'extraits et fractions de Borassus aethioplum Mart (Arécaceae) approche du mécanisme biochimique d'action. Thèse Unique en Sciences biologiques appliquées. Université de Ouagadougou, p. 214. 